\title{
EDUCACIÓN Y GESTIÓN EN AMÉRICA LATINA
}

José Solano Alpízar ${ }^{1}$

\section{Resumen}

El presente artículo constituye un ejercicio descriptivo acerca del tema de la gestión educativa en América Latina. El mismo ofrece una visión general del contexto socio-histórico en que ésta ha tenido lugar.

En primera instancia, se sitúa el modelo de desarrollo diseminado por la CEPAL en América Latina, a partir de la segunda mitad del siglo XX y sus implicaciones en la configuración del modelo educativo adoptado en la región por los diferentes países.

Posteriormente, hace una revisión del tránsito experimentado por el proceso de gestión educativa durante las décadas subsiguientes; por último, presenta una breve caracterización de las transformaciones más significativas que ha experimentado la gestión educativa de cara a los desafíos que se vislumbran con el ingreso al siglo XXI.

\section{Abstract}

Descriptive exercise that addresses the topic of Education Management in Latin America. It gives a general view of the socio-historical context in which this management has taken place.

The article first presents the development model spread by CEPAL (Spanish acronym for the Economic Commission of the UN for Latin America and the Caribbean) in Latin America after the second half of the twentieth century and its influence in shaping the educational model adopted by different countries of the region.

Then, we review the road traveled by the educational management process during the following decades. Finally, there is a brief description of the most important transformations this management has had after the challenges encountered during the beginning of the twenty-first century.

Descriptores: educación, gestión, pedagogía. sociedad

\footnotetext{
${ }^{1}$ José Solano Alpízar. Historiador y Pedagogo con Especialidad en Investigación Educativa. Se desempeñó como Coordinador del Programa de Investigación Educativa del Centro de Investigación y Docencia en Educación. Universidad Nacional. Actualmente, es el Director de Docencia.
} 

ablar de gestión educativa es hablar necesariamente sobre un tema en el que América Latina ofrece una amplia y rica experiencia que se remonta a los inicios de la década del cincuenta. Contexto en el que comenzó a perfilarse lo que habría de conocerse en adelante como paradigma de la "Educación para el desarrollo"2, donde la gestión educativa pasó a ocupar un espacio sensible en la determinación de las políticas educativas nacionales y en el cual asumieron un papel muy importante las recomendaciones emanadas de organismos internacionales y regionales como la UNESCO y la CEPAL. A partir de este momento, se le confirió a la educación el papel de herramienta fundamental para el logro del desarrollo económico-social de los países latinoamericanos; pasó a ser concebida como vehículo de movilidad social capaz de permitir a los individuos ascender en la estructura social y tener la posibilidad de lograr altos rendimientos por la inversión en su propia educación ${ }^{3}$.

La preocupación de los diversos gobiernos del área por expandir la cobertura del sistema educativo, sometió la gestión escolar a una visión normativa y planificadora muy consecuente con las ideas "desarrollistas" adoptadas por los gobiernos y sus respectivos Ministerios de Educación y Planificación, a quienes los organismos de asistencia financiera les exigían el establecimiento de planes de desarrollo y una programación apropiada. Como el énfasis en este periodo estuvo centrado en la cobertura educativa, se le dio menos importancia a la calidad del conocimiento, al cómo educar, al quién educar y al para qué educar. Este excesivo énfasis en la cobertura estuvo vinculado con la fe en el mito del "desarrollo económico" para el que la educación cumplía un papel determinante, ya que contemplaba el aumento permanente de la matrícula en el sistema educativo formal y un constante incremento de las asignaciones de fondo públicos, aunado a reformas del contenido y la distribución de la educación (Marshall, 1981).

En las décadas del sesenta y el setenta, aunque la educación siguió siendo concebida como el principal vehículo de movilidad social, la gestión adquirió matices distintos a los conocidos en la década del cincuenta, pues se comenzaron a introducir elementos de futuro en la planificación y, consecuentemente, en la gestión; estableciéndose una planificación con criterio "prospectivo" que tomaba en consideración las necesidades de recursos humanos, mapas escolares y micro planificación, entre otras. En este contex to, tuvieron fuerte incidencia las teorías del capital humano; particularmente los planeamientos de Thrurow,

\footnotetext{
2 Un análisis detallado sobre la "Educación para el desarrollo" se puede encontrar en el libro de mi autoría: Educación y Desarrollo en América Latina: Un análisis histórico-conceptual, Heredia: EUNA. 2001.

${ }_{3}$ Medina, Echavarría. José (1979). Filosofía, Educación y Desarrollo. México: Siglo XXI.
} 
Schultz y Becker acerca de la educación como inversión, que fueron bien recibidos por los gobiernos latinoamericanos que comenzaron a destinar mayor cantidad de recursos a la educación.

No es casual entonces que al cierre de la década del setenta, se presente en la región un modelo de gestión educativa influido por los estudios comparativos, modelos estrategias, y con un claro predominio -a nivel metodológicodel análisis de costo-beneficio en la toma de decisiones sobre opciones y proyectos alternativos. Posteriormente en la llamada "década perdida" -los años ochenta- se acentuó esta tendencia, según la cual se debían vincular las consideraciones económicas con la planificación y la gestión.

Este estilo gestionario se vio favorecido, en gran parte, debido a las condiciones que propició el problema del pago de la deuda externa, ya que ésta trajo consigo la reducción de presupuestos en actividades sociales de peso como salud y educación, situación que obligó a los gobiernos a programar presupuestariamente, de acuerdo con el contexto de crisis en que se vivía. La reducción experimentada por los servicios sociales en esta década, se aprecia con claridad en el ámbito educativo, tanto en sus aspectos pedagógicos -trabajo de aulacomo en la profundización del deterioro de la infraestructura educativa, y aunque en términos de cobertura se siguió haciendo un esfuerzo sostenido, los niveles de inequidad en cuanto a las posibilidades de acceso se incrementaron, debido a las condiciones más generales de deterioro salarial y baja en los niveles de vida de los sectores menos favorecidos económica y socialmente.

Este deterioro se vio reflejado en el significativo incremento de los índices de repetición y fracaso escolar -especialmente en la educación básica- la interrupción del proceso de incorporación de los hijos de familias de sectores populares a los niveles post-básicos del sistema educativo, el aumento en la diferenciación interna del sistema educativo, el deterioro en la calidad de la educación y la mayor concentración gubernamental en los esfuerzos coyunturales por sobre la proyección de mediano y largo plazo ${ }^{4}$.

Este contexto de crisis que tuvo como epicentro la década de los ochenta, abrió el espacio para que se generara una dinamización en las estrategias de las políticas públicas; se reconoció a la escuela como uno de los factores determinantes en los resultados del proceso educativo, y a la educación, como un medio para la transformación productiva con equidad y la promoción de una ciudadanía afirmada en valores democráticos, de solidaridad, participación y concertación social..$^{5}$ A su vez, el proceso de transnacionalización de las economías

\footnotetext{
${ }^{4}$ Tedesco, Juan Carlos (1992) "La gestión en la encrucijada de nuestro tiempo". En: Ezpeleta Justa y Alfredo Furlan (Comp.). La gestión pedagógica de la escuela. Santiago de Chile: UNESCO/OREALC.

${ }^{5}$ Al respecto véase: CEPAL/UNESCO (1992). Educación y Conocimiento: Eje de la transformación productiva con equidad. Santiago de Chile: CEPAL/UNESCO.
} 
generó un marco propicio para un discurso sobre el desarrollo que se vino a afirmar en la tesis de la imposibilidad latinoamericana de permanecer al margen de la globalización, asentándose el terreno para el retorno de las viejas teorías del capital humano que predominaron en los sesentas y en las que se apostaba fuertemente a la educación como pivote del desarrollo económico.

Para satisfacer los requerimientos del nuevo paradigma productivo, en el plano de la educación, se plantea la necesidad de incorporar innovaciones científicas y tecnológicas en el currículum escolar, lo que viene a redundar en la necesidad de formar recursos humanos con
Este estilo gestionario se vio favorecido, en gran parte, debido a las condiciones que propició el problema del pago de la deuda externa, ya que ésta trajo consigo la reducción de presupuestos en actividades sociales de peso como salud y educación, situación que obligó a los gobiernos a programar presupuestariamente, de acuerdo con el contexto de crisis en que se vivía.

una mayor capacidad productiva y competitiva, a la vez que con gran disponibilidad al cambio en materia de formación y capacitación laboral.

La escuela y la educación se vinieron a reposicionar en el centro de un discurso que promovía la transformación productiva con equidad a partir de una redefinición del papel de las instituciones escolares al interior de la sociedad; todo ello coincidió, en líneas muy generales, con los planeamientos sobre descentralización, autonomía, participación social en las políticas educativas, que venían escuchándose desde finales de la década del setenta y que le apostaban a la escuela y al colectivo que convive en ella como el lugar privilegiado del cual podría partir cualquier transformación, así como desde donde se podría promover el mejoramiento de la calidad educativa.

\section{La Gestión Educativa: una mirada reciente}

La década del ochenta convierte a la escuela en el núcleo sobre el que se dirigirán todas las miradas. Ello debido, en gran medida, al agotamiento que mostraban los discursos sobre el cambio y el mejoramiento de la calidad educativa de manera vertical; es decir, los cambios por decreto desde las instancias de poder decisional, así como por el constante fracaso de las políticas ministeriales dirigidas al docente (capacitación, materiales, metodologías didácticas, y 
otros). Aunado a ello, debemos mencionar el surgimiento de una preocupación en América Latina por la identidad de la institución o modelo de gestión escolar en el que las ideas de autonomía, descentralización y capacidad en la toma de decisiones, adquieren una importancia creciente.

El leve impacto de las políticas de descentralización para mejorar la calidad de la educación durante las década del setenta y el ochenta, generó la necesidad de construir hipótesis acerca de considerar a la escuela como "locus privilegiado" de la intervención, lo cual ha ayudado al desarrollo de nuevos modelos de gestión institucional. En ello han tenido una participación destacada las instancias gubernamentales, que han realizado un esfuerzo por dinamizar el proceso educativo, para lo cual se han venido creando marcos legales y normativos relacionados con los procesos de descentralización educativa y de participación social. ${ }^{6}$

Lo anterior ha traído aparejado consigo el despertar de expectativas tanto en las comunidades como en las instituciones educativas respecto a la autonomía y a la capacidad de las propias escuelas por generar proyectos educativos adecuados a sus condiciones. Hoy por hoy, se habla de la necesidad de crear un nuevo tipo de escuela, donde prevalezca un estilo de gestión caracterizado -entre otros aspectos- por una inclinación firme de los gestores hacia los aspectos educativos en correspondencia con condiciones particulares de la escuela, y para establecer una traducción propia de la normatividad y la necesidad de contar con procesos de evaluación para el cambio institucional.

Este nuevo estilo de gestión implica la aceptación de que cada escuela tiene su propia identidad y que las dinámicas a su interior responden a la particularidad de las relaciones que se establecen en el colectivo. El trabajo en equipo constituye parte medular en este esquema, en la medida en que el esfuerzo desarrollado por el responsable de la institución -en este caso el director o directora- es compartido por el resto de miembros de la institución. La aceptación del entorno y la apertura a las influencias, exigencias y demandas de éste, ocupa otro espacio importante en la configuración del nuevo estilo de gestión, por cuanto sólo contemplando la participación de la comunidad (padres, madres, grupos comunales, empresarios, intelectuales, entre otros) puede la escuela dejar de ser una ínsula y adquirir un rendimiento que le permita alimentar y retroalimentarse del medio que lo rodea.

La posibilidad de que la institución pueda destinar los recursos asignados para canalizarlos de acuerdo con su propio proyecto educativo, es una condición necesaria para el logro eficaz de los objetivos del mismo. A la vez que se

${ }^{6}$ Sobre normativas y marcos legales para los procesos de gestión pueden analizarse más a profundidad el caso chileno y el mexicano. 
hace inminente la necesidad de que la escuela rinda cuentas acerca de su accionar, dado que es un servicio público y como tal debe cumplir con las demandas y exigencias de los beneficiarios y usuarios del mismo.

De esta manera puede decirse que los ejes que cruzan la concepción de un nuevo estilo de gestión son:

- AUTONOMÍA

- DESCENTRALIZACIÓN

- LIDERAZGO (orientado a lo académico)

- NORMATIVIDAD (reducción)

- CONTROLES EXTERNOS (reducción)

- $\quad$ ASIGNACIÓN Y DESTINO DE RECURSOS

- IDENTIDAD INSTITUCIONAL

- $\quad$ RENDIMIENTO DE CUENTAS

- EVALUACIÓN EXTERNA

- ATENCIÓN A RESULTADOS (focalizar)

- $\quad$ PARTICIPACIÓN COMUNITARIA

- MEJORAMIENTO DE LA CALIDAD EDUCATIVA

\section{La Gestión: sus niveles de concreción}

El interés actual de la gestión en el ámbito educativo se centra en "la escuela" como totalidad en la cual confluyen y se materializan diversos elementos de los diferentes niveles de gestión, a saber: la educativa, la escolar y la pedagógica.

En relación con los elementos identificados de la gestión educativa, destacan las maneras en que se pueden organizar los sistemas educativos, es decir, aquellas formas que se caracterizan por las atribuciones e iniciativas del Estado. Las principales funciones que se cumplen son las financieras, administrativas y pedagógicas. Estas se traducen en formulación de políticas educativas, estudio y planificación de necesidades de recursos humanos, escuelas y plazas docentes, así como organización y graduación de los niveles de responsabilidad, de coordinación de actividades y de evaluación de resultados de subsistemas y sus instituciones.

Entre los elementos sobresalientes de la política educativa del sector, aparecen los procesos de descentralización, legislación y reformas en contenidos curriculares. La primera en la medida que ha hecho responsable de los planteles educativos a las comunidades en términos de sus recursos materiales, humanos y financieros; la segunda, porque es a partir de ésta que se determina 
La reducción experimentada por

los servicios sociales en esta década, se aprecia con claridad sus aspectos pedagógicos -trabajo de aula - como en la profundización del deterioro de la infraestructura educativa, y aunque en términos de cobertura se siguió haciendo un esfuerzo sostenido, los niveles de inequidad en cuanto a las posibilidades de acceso se incrementaron, debido a las condiciones más generales de deterioro salarial y baja en los niveles de vida de los sectores menos favorecidos económica y socialmente. en el ámbito educativo, tanto en

la normatividad vigente para todos y cada uno de los planteles en la cual se circunscribirá la tarea educativa y la tercera, porque establece un nuevo currículum. Asimismo en este nivel, de manera paralela, se inscriben las figuras de las organizaciones gremiales magisteriales que participan en la determinación de las políticas educativas nacionales y locales, así como la participación de la sociedad civil a través de organizaciones comunales, padres de familia, empresarios, organizaciones no gubernamentales, intelectuales, entre otras.

Un mayor número de elementos se inscriben en la gestión escolar por ser este el espacio en el cual se concreta tanto la política educativa como los elementos que corresponden a la dinámica interna del centro escolar. En este nivel, quedan incluidos simultáneamente, aunque con diferente peso, las dimensiones organizacionales, pedagógicas, laborales, administrativas y sociales en el plano cotidiano para poner en acto la prestación del servicio educativo, en donde la interacción de los sujetos en la conformación de los procesos le brindan una caracterización particular a los componentes.

En este sentido, cabe decir que la gestión escolar constituye ese conjunto de acciones relacionadas entre sí que emprende el colectivo de la institución, y en el que el liderazgo del responsable formal de la institución juega un papel importante, a la vez que debe ser comprendido éste, como ese espacio donde se cruzan diferentes proyectos y programas generados por los sujetos que participan y que no son solamente los de los maestros y la dirección, sino que contempla a los de los padres de familia, alumnos y a la comunidad misma.

La gestión pedagógica constituye, por su parte, el entramado de lo organizacional, el currículo formal y el currículo oculto. En ella intervienen particularmente 
el sistema de evaluación oficial, las propuestas de los maestros, las indicaciones generales para la disciplina de la escuela desde la normativa, y las diferentes medidas locales y personales puestas en juego en el centro escolar. Como bien lo plantea Ezpeleta (1992) "es un concepto que se centra en el espacio de la escuela, que es su condición de posibilidad y su ámbito de excelencia. La estructura y dinámica organizativa de la escuela, su conformación profesional técnica y material constituyen las bases reales para que el currículo y su operación encuentren una forma determinada" (p. 107).

En este nivel y ubicando un plano operativo, puede decirse que la gestión se constituye a partir de las relaciones que se construyen entre alumnos y maestros, entre maestros y directivos, entre directivos y alumnos, y de las dinámicas que se llevan a cabo dentro del aula y que explican los procesos de aprendizaje de lo normado y de lo que no lo está. Implica el manejo disciplinario, las concepciones de aprendizaje, de enseñanza y de evaluación, así como el ordenamiento físico del aula. También significa el cruce de las historias personales de los sujetos, el conjunto de costumbres, hábitos, prácticas y tradiciones construidas a partir del proceso interactivo y de las identidades que establecen un perfil específico a la institución.

A manera de cierre provisional, podríamos decir que los espacios de acción e interacción, de independencia e influencia entre estos niveles de la gestión, no se encuentran ni pueden encontrarse perfectamente delineados. No se puede hablar de que existen fronteras que delimiten al uno del otro y viceversa, pues se produce una relación dialéctica en el que cada uno imprime rasgos que le son propios, y donde los cambios que se producen en uno van configurando las características del otro. Las formas en que estos niveles se articulan dependen-claro está- de las condiciones nacionales, locales y particulares que en un momento determinado configuran el fenómeno educativo, así como de la participación de los sujetos y las formas en que se dan los procesos.

Los diferentes tipos de gestión que se han configurado, educativa, escolar, pedagógica, se vinculan tanto a razones de tipo histórico como a formas de trabajo en ámbitos específicos: sistema educativo, plantel o aula. Cada uno de los múltiples enfoques destaca un determinado tipo de relación que se establece entre los sujetos mismos y entre estos y el contexto, según el posicionamiento desde donde se intente explicar la serie de prácticas articuladoras entre el discurso y el actuar cotidiano. De manera tal que la gestión como concepto y como herramienta vinculada a procesos específicos, está en construcción. Las aportaciones de la investigación educativa para explicar y reconocer algunas de las experiencias aplicadas en casos concretos, han contribuido a dimensionareste complejo campo y de hecho vendrán a nutrir un acervo de conocimientos más preciso sobre las dimensiones que envuelven la gestión educativa en América Latina. 


\section{REFERENCIAS}

CEPAL (1990). La transformación productiva con equidad. La tarea prioritaria de América Latina y el Caribe. Santiago: CEPAL.

CEPAL/UNESCO (1992). Educación y Conocimiento: Eje de la transformación productiva con equidad. Santiago: CEPAL/UNESCO.

Ezpeleta Justa y Alfredo Furlan (Comp.). La gestión pedagógica de la escuela. Santiago: UNESCO/OREALC.

Medina, Echavarría, José (1979). Filosofía, Educación y Desarrollo. México: Siglo XXI.

Solano, J. (2001). Educación y Desarrollo en América Latina: Un análisis históricoconceptual, Heredia: EUNA.

Tedesco, J. (1992). "La gestión en la encrucijada de nuestro tiempo". En: Ezpeleta Justa y Alfredo Furlan (Comp.). La gestión pedagógica de la escuela. Santiago de Chile: UNESCO/OREALC.

Wolfe, M. (1981). “Estilos de desarrollo y educación: Un inventario de mitos, recomendaciones y potencialidades". Revistas de la CEPAL, $\mathrm{N}^{\circ} 21$. Santiago: CEPAL. 